International Journal of Modern Physics B

Vol. 34, Nos. 14-16 (2020) 2040084 (8 pages)

(C) World Scientific Publishing Company

DOI: $10.1142 / \mathrm{S} 0217979220400846$

\title{
Rebuilding experimental nonequilibrium radiation in shock-heated Martian-like mixture flows using electronic state-to-state approach
}

\author{
Qizhen Hong*,†, Xiaoyong Wang*, Yuan $\mathrm{Hu}^{*}$, Xin Lin* and Quanhua Sun*,†, \\ * State Key Laboratory of High Temperature Gas Dynamics, \\ Institute of Mechanics, Chinese Academy of Sciences, \\ Beijing 100190, P. R. China \\ ${ }^{\dagger}$ School of Engineering Science, University of Chinese Academy of Sciences, \\ Beijing 100049, P. R. China \\ ¥qsun@imech.ac.cn
}

Received 15 September 2019

Accepted 15 February 2020

Published 30 May 2020

\begin{abstract}
An electronic state-to-state approach is developed to reproduce numerically the radiative processes in the experiments on the Electric Arc Shock Tube facility at NASA Ames Research Center. The experiments measured the spectral radiance of $\mathrm{C}_{2}$ Swan band and $\mathrm{CO}$ 4th Positive band behind the strong shock wave in a Martian-like mixture. The present state-to-state approach solves the electronic states of strong radiators by integrating the collisional-radiative model into the master equations in the frame of Euler equations. Particularly, the electron impact dissociation of $\mathrm{CO}$ is included, and the rate coefficients are proposed for the electronic state-specific heavy-particle impact excitation and dissociation of $\mathrm{C}_{2}$. The nonequilibrium radiation behind the shock is calculated by the line-by-line method, and it is then convoluted using the calibrated smearing function in order to compare with the experiments. The state-specific simulation results is found to agree well with both spectral and spatial measurement data. The simulated electronic state populations of $\mathrm{CO}$ and $\mathrm{C}_{2}$ deviate from Boltzmann distributions significantly, which could explain the failure of previous two-temperature quasi-steady-state based simulations.
\end{abstract}

Keywords: Electronic state-to-state approach; nonequilibrium radiation; Martian-like mixture.

PACS numbers: 47.40.Ki, 47.40.-x, 47.70.Nd

‡Corresponding author. 


\section{Introduction}

The atmospheric entry velocity for aerocapture missions to Mars is estimated to be $6-9 \mathrm{~km} / \mathrm{s}$, with radiation contributing to more than $80 \%$ of the total heat flux at velocities over $8.5 \mathrm{~km} / \mathrm{s} .{ }^{1}$ The traditional approach to studying such kind of flows involves the multi-temperature models where the quasi-steady-state (QSS) assumption is employed and empirical parameters are calibrated using available validated data. ${ }^{2}$ However, the physically accurate state-to-state approach ${ }^{3}$ revealed the invalidity of the QSS assumption. ${ }^{4}$ Particularly, the population of the $\operatorname{CO}\left(A^{1} \Pi\right)$ state, which is critical for the CO 4th Positive band, is significantly underestimated by the Boltzmann distribution. $\underline{4}$

Recently, the nonequilibrium radiation for shock-heated CO flows has been measured in the Electric Arc Shock Tube (EAST) facility at NASA Ames Research Center. .5 Cruden and his collaborators presented an analysis of $\mathrm{CO}$ and $\mathrm{C}_{2}$ radiation using a two-temperature QSS-based (2T-QSS) $\operatorname{method}^{6}$ and showed that the spectrum of $\mathrm{CO}$ vacuum ultraviolet (VUV) radiation does not match well with the measurement. The radiation peak in the $\mathrm{C}_{2}$ Swan band is always over-predicted due to the adoption of the Boltzmann equilibrium model therein. $\frac{6}{}$

This work aims to improve the accuracy of numerical prediction on the nonequilibrium radiation in the shock-heated Martian-like flows. To overcome the deficiency of the 2T-QSS method, we have developed an electronic state-to-state approach, which is capable of capturing the strong nonequilibrium effects. The present numerical results are compared with the measurements from EAST facility, showing a significant improvement is achieved by the electronic state-to-state approach.

\section{Nonequilibrium Thermochemical Modeling}

\subsection{Species in state-to-state approach}

Seventeen shots (shots 90-106) for pure CO flows have been carried out in EAST $^{5}$ at an initial pressure of 0.25 Torr, where the most significant radiative signatures from $\mathrm{C}_{2}$ Swan band and CO 4th Positive band were measured. In order to rebuild the nonequilibrium radiation measured in those experiments, the mixture is modeled using the following species in the present numerical calculation: $\mathrm{CO}_{2}, \mathrm{CO}, \mathrm{O}_{2}$, $\mathrm{C}, \mathrm{O}, \mathrm{C}_{2}, \mathrm{C}^{+}, \mathrm{O}^{+}, \mathrm{O}_{2}^{+}, \mathrm{CO}^{+}$and $\mathrm{e}-$. Among them, the electronic states of strong radiators are treated as separate pseudo-species (i.e., $\mathrm{CO}\left(X^{1} \Sigma^{+}, a^{3} \Pi, a^{\prime 3} \Sigma^{+}, d^{3} \Delta\right.$, $\left.e^{3} \Sigma^{-}, A^{1} \Pi\right), \mathrm{C}_{2}\left(X^{1} \Sigma_{g}^{+}, a^{3} \Pi_{u}, b^{3} \Sigma_{g}^{-}, A^{1} \Pi_{u}, c^{3} \Sigma_{u}^{-}, d^{3} \Pi_{g}\right), \mathrm{O}\left({ }^{3} P,{ }^{1} D,{ }^{1} S\right)$ and $\left.\mathrm{C}\left({ }^{3} P,{ }^{1} D,{ }^{1} S,{ }^{5} S^{0}\right)\right)$ to be solved using the electronic state-to-state approach. For other species, only the ground electronic state is modeled due to their small contribution to radiation.

\subsection{Electronic state-specific rate coefficients}

The molecular electronic state populations are modeled using a collisionalradiative model, $\underline{3}$ which accounts for the repopulation and depopulation of the 
Table 1. Rate coefficients for $\mathrm{C}_{2}$ electronic state-specific dissociation proposed in this paper.

\begin{tabular}{cccc}
\hline Electronic state & $A\left(\mathrm{~cm}^{3} / \mathrm{s}\right)$ & $n$ & $E_{a}(\mathrm{~K})$ \\
\hline $\mathrm{C}_{2}\left(X^{1} \Sigma_{g}^{+}\right)$ & $1.93 \times 10^{-11}$ & 0.462 & 64231.1 \\
$\mathrm{C}_{2}\left(a^{3} \Pi_{u}\right)$ & $2.25 \times 10^{-11}$ & 0.458 & 63243.7 \\
$\mathrm{C}_{2}\left(b^{3} \Sigma_{g}^{-}\right)$ & $2.82 \times 10^{-11}$ & 0.441 & 54712.4 \\
$\mathrm{C}_{2}\left(A^{1} \Pi_{u}\right)$ & $2.77 \times 10^{-11}$ & 0.432 & 52072.3 \\
$\mathrm{C}_{2}\left(c^{3} \Sigma_{u}^{-}\right)$ & $2.34 \times 10^{-11}$ & 0.425 & 44826.3 \\
$\mathrm{C}_{2}\left(d^{3} \Pi_{g}\right)$ & $5.54 \times 10^{-11}$ & 0.337 & 35362.1 \\
\hline
\end{tabular}

electronic states through collisional (including excitation $\backslash$ de-excitation, dissociation $\backslash$ recombination and ionization by electron and heavy-particle impact) and radiative (bound-bound transition) processes. However, the electronic state-specific rate coefficients of $\mathrm{C}_{2}$ are unavailable as related reactions have not been considered previously. In this work, the heavy-particle impact excitation rate coefficients of $\mathrm{C}_{2}$ are calculated following the method of Park. ${ }^{7}$ The heavy-particle impact dissociation rate coefficients of $\mathrm{C}_{2}$ are proposed (listed in Table 1 using the modified Arrhenius form) using the generalized Treanor-Marrone method. ${ }^{9}$

The electronic state-specific heavy-particle impact dissociation rate coefficients of $\mathrm{CO}$ are assessed by using two different datasets of parameters taken from Johnston et al. ${ }^{10}$ and Park, ${ }^{7}$ respectively. The effects on radiation of these two models are examined in Sec. 3.2. For the rest of the kinetic processes (the electron impact dissociation of $\mathrm{CO}$ is also included), the state-specific rate coefficients proposed by Johnston et al..$^{10}$ are utilized, except that the 'optically variable' model proposed by Potter $^{8}$ is used for the radiative processes with the wavelength-dependent escape factor.

\subsection{Numerical modeling}

The one-dimensional nonequilibrium Euler equations are employed to compute the post-shock flow, and the master equations are integrated to calculate the species concentration.

Totally, 26 electronic states (as mentioned in Sec. 2.1) of different species and 159 state-specific reactions are considered in the master equations. The temperatures $T_{\mathrm{tr}}$ and $T_{v}$ are used to account for the translational-rotational energy and vibrational energy, respectively. The vibrational energy transfer terms are modeled in the same manner as Ref. 4. The post-shock initial conditions are evaluated using the Rankine-Hugoniot relations assuming the internal energy modes to be frozen. The governing equations are numerically solved in a full couple manner using the Backward-Differentiation-Formula method.11

\subsection{Radiation modeling}

As for the pure $\mathrm{CO}$ shock-heated flows of EAST shots, the 4th Positive band $\left(\mathrm{CO}\left(A^{1} \Pi\right)-\mathrm{CO}\left(X^{1} \Sigma^{+}\right)\right)$in the VUV dominates the radiation feature of $\mathrm{CO}$, and 
the primary radiation feature of $\mathrm{C}_{2}$ comes from the Swan band, i.e., $\mathrm{C}_{2}\left(d^{3} \Pi_{g}\right)-$ $\mathrm{C}_{2}\left(a^{3} \Pi_{u}\right)$, which dominates in the 'Red' spectral region. Radiations of these two bands are simulated using the line-by-line radiation code (Photaura ${ }^{8}$ ) with up-to-date electronic-vibrational transition moments for diatomic bound-bound transition. The radiation-flowfield coupling is implemented by assuming 'optically thick' for VUV radiation and 'optically thin' for other spectral regions. Post-shock spatial distribution of the spectrally resolved radiance (monochromatic intensity) can be calculated as follows:

$$
I(x, \lambda)=S(x, \lambda)[1-\exp (-k(x, \lambda) D)]
$$

where $\lambda$ is wavelength, $x$ is the position in shock tube, $S$ is the source function, $k$ is the absorption coefficient and $D$ denotes the diameter of shock tube.

\section{Results}

\subsection{Radiation data rebuilding}

In the measurement, various factors can affect the spectral and spatial resolution of the measured radiance, including the resolution of optical setup, charge sharing of the spectrometer and shock motion during the camera acquisition. In order to make comparison between simulated and measured radiance of EAST shots, the raw simulation results are convoluted using the total smearing function proposed by Cruden et al. where the instrumental line shape and the spatial resolution function are included and calibrated. ${ }^{6}$ One example of convolution effect is shown in Fig. 1 for shot 100 .

\subsection{Comparison between simulation and experiment}

The present electronic state-to-state approach is examined by comparing both simulated emission radiances of $\mathrm{C}_{2}$ and $\mathrm{CO}$ over the range of velocities from $3.4-9.5 \mathrm{~km} / \mathrm{s}$
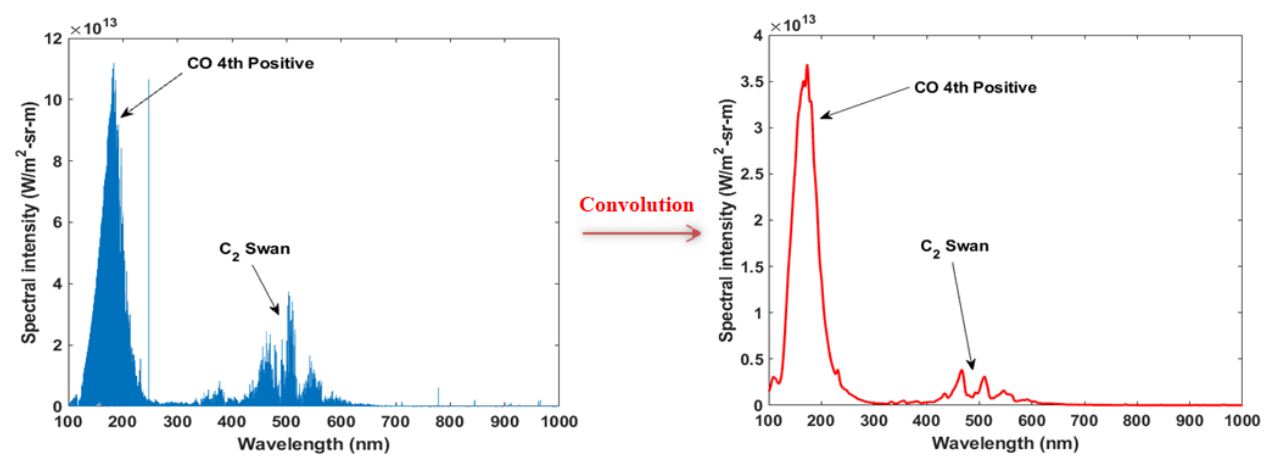

Fig. 1. (Color online) Simulation results of spectral intensity in EAST shot 100. Left: raw simulation results; Right: convoluted simulation results. 

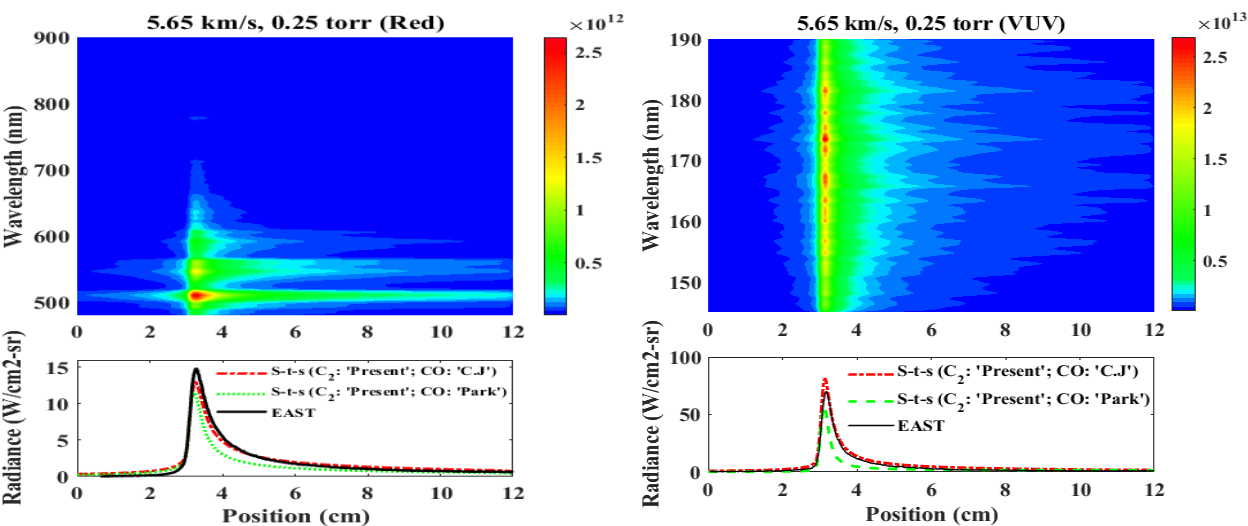

Fig. 2. (Color online) Simulated radiance versus position and wavelength (upper) and the comparison of spatial radiance between simulation and measurement (low). Left: $\mathrm{C}_{2}$ Swan band (480900 nm); Right: CO 4th Positive band (145-190 nm).

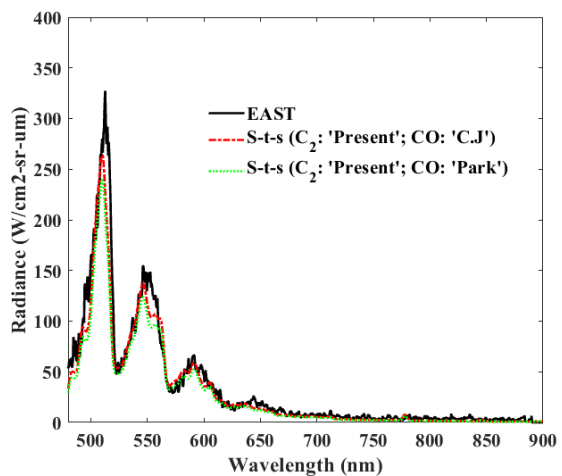

(a) $0 \mathrm{~cm}$ after the location of peak radiance

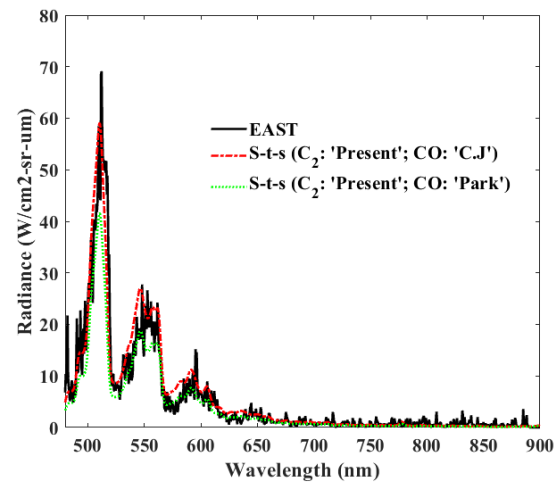

(c) $2 \mathrm{~cm}$ after the location of peak radiance

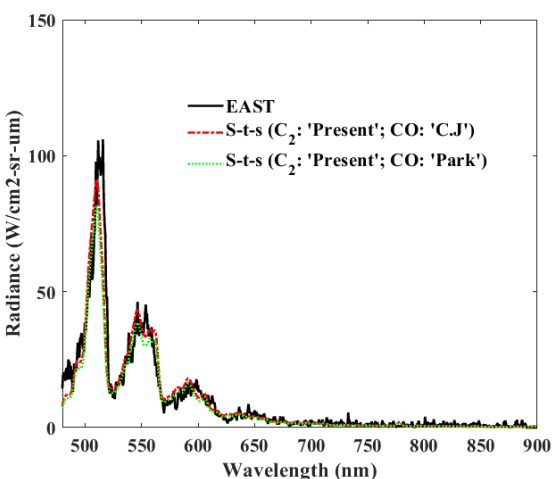

(b) $1 \mathrm{~cm}$ after the location of peak radiance

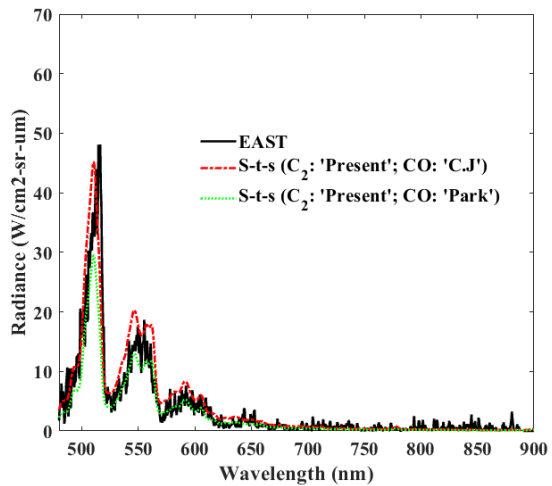

(d) $3 \mathrm{~cm}$ after the location of peak radiance

Fig. 3. (Color online) Comparison of spectral radiance (480-900 nm) between state-to-state calculations and measurements at different positions behind the shock for the case of shot 100 $(5.65 \mathrm{~km} / \mathrm{s}, 0.25$ Torr). Positions correspond to $0,1,2$ and $3 \mathrm{~cm}$ after the location of peak radiance. 
(shots 90-106 in EAST) with experimental measurements. Figure 2 presents a colormap of the absolute spectral and spatial radiance for one shot (shot 100 is shown as an example). Clearly, using the $\mathrm{CO}$ electronic state-specific dissociation rate coefficients proposed by Johnston et al. ${ }^{10}$ with the present state-specific rate coefficients of $\mathrm{C}_{2}$ (namely [ $\mathrm{C}_{2}$ : 'present'; $\mathrm{CO}$ : 'C.J'] in Fig. 2) predicts better than using Park's $\mathrm{CO}$ electronic state-specific dissociation rate coefficients ${ }^{7}$ with present state-specific rate coefficients of $\mathrm{C}_{2}$ (namely $\left[\mathrm{C}_{2}\right.$ : 'present'; $\mathrm{CO}$ : 'Park'] in Fig. 2) in both radiation bands of shot 100. In addition, it is observed from the results of all 17 shots that using Johnston's dissociation rate coefficients ${ }^{10}$ for CO underpredict the dissociation rate in the lower velocity range but agrees well at higher velocity (larger than $4.4 \mathrm{~km} / \mathrm{s}$ ), it implies that different rate models for CO reactivity should be employed at different velocity regimes, which is also suggested by Cruden et al. 6

Figure 3 shows the comparison of $\mathrm{C}_{2}$ spectral radiance at four different positions behind the shock for the case of shot 100. It is seen that the results obtained using the present rate coefficients of $\mathrm{C}_{2}$ agree excellently with the observed $\mathrm{C}_{2}$ ra-

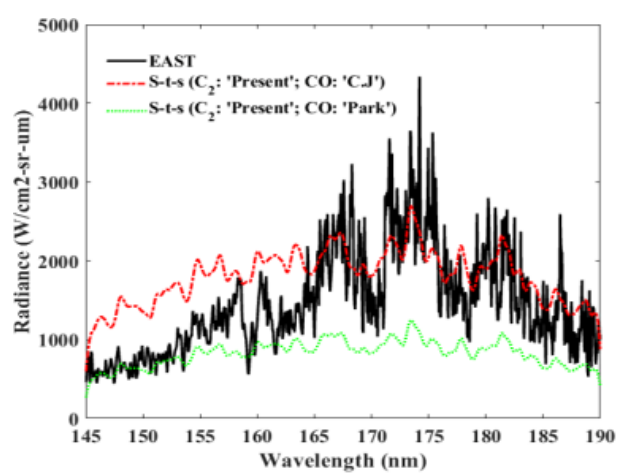

(a) $0 \mathrm{~cm}$ after the location of peak radiance

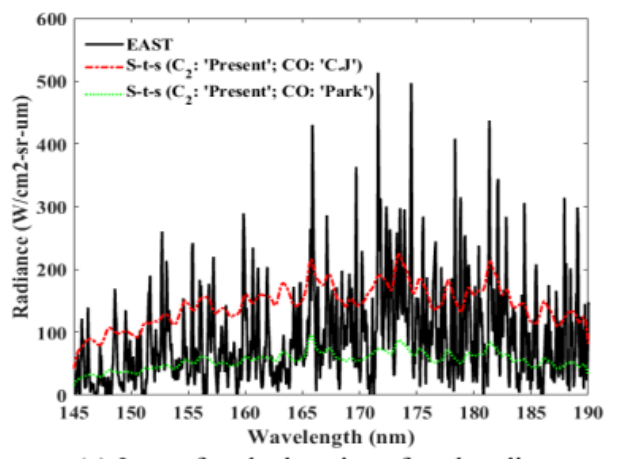

(c) $2 \mathrm{~cm}$ after the location of peak radiance

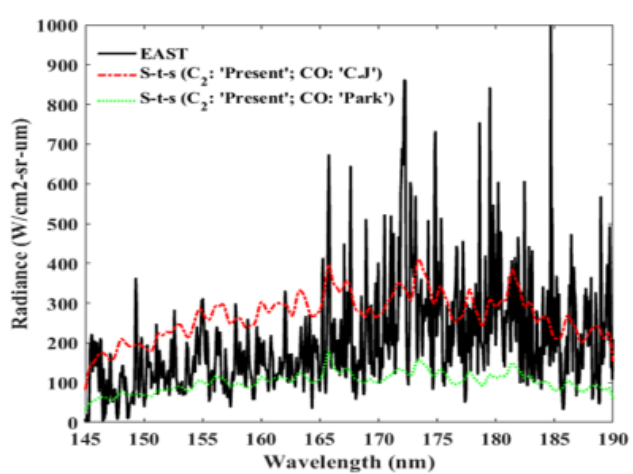

(b) $1 \mathrm{~cm}$ after the location of peak radiance

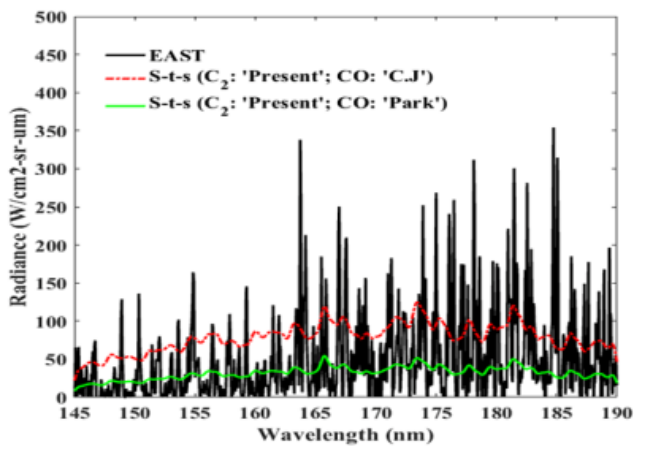

(d) $3 \mathrm{~cm}$ after the location of peak radiance

Fig. 4. (Color online) Comparison of spectral radiance (145-190 nm) between state-to-state calculations and measurements at different positions behind the shock for the case of shot 100 $(5.65 \mathrm{~km} / \mathrm{s}, 0.25$ Torr). Positions correspond to $0,1,2$ and $3 \mathrm{~cm}$ after the location of peak radiance. 
diance regardless of the model for $\mathrm{CO}$, which validates the present rate coefficients for $\mathrm{C}_{2}$. Since using the 2T-QSS assumption does not match well spectrally with the measurement in CO VUV radiation and overpredicts the radiation peak in the $\mathrm{C}_{2}$ Swan band, $\frac{6}{-}$ the good agreement achieved in present simulation denotes that the electronic state populations of $\mathrm{CO}$ and $\mathrm{C}_{2}$ based on present electronic stateto-state approach are more accurate, which are actually deviated from the Boltzmann distribution significantly. Figure 4 shows the CO spectral radiance. Again, the agreement between simulation and measurement is satisfactory and the differences are all within the experimental error. It is also seen that using Park's electronic state-specific dissociation rate coefficients of $\mathrm{CO}^{7}$ under-predicts the radiance than using Johnston's rate coefficients ${ }^{10}$ within the whole spectral range, because CO dissociation rate estimated by Park's rate coefficients is larger.

\section{Conclusions}

An electronic state-to-state approach for pure CO shock heated flows is developed in this paper and the state-specific heavy-particle impact excitation and dissociation rate coefficients of $\mathrm{C}_{2}$ are proposed. The nonequilibrium $\mathrm{C}_{2}$ Red radiation and CO VUV radiation calculated by the present model agree well with the experimental measurement of EAST shots for both the peak radiance and radiation profile. It is found that different rate models for CO dissociation should be employed at different velocity regimes. Detailed electronic state populations of $\mathrm{CO}$ and $\mathrm{C}_{2}$ obtained by present model are more accurate, which are different from the results obtained by 2T-QSS method.

\section{Acknowledgments}

This work was supported by the Strategic Priority Research Program of Chinese Academy of Sciences (Grant No. XDA17030100). The authors would like to thank Dr. Daniel Potter for the useful discussion. We also gratefully acknowledge Dr. B. A. Cruden for providing the experimental data in the internet.

\section{References}

1. R. D. Braun, R. W. Powell and L. C. Hartung, Technical Paper 3019, NASA Langley Research Center, September 1990.

2. C. Park, Nonequilibrium Hypersonic Aerothermodynamics, 1st edn. (Wiley, 1990).

3. S. M. Jo, O. J. Kwon and J. Kim, in AIAA Scitech 2019 Forum, 0799 (2019).

4. R. L. Macdonald et al., Phys. Rev. Fluids 1, 043401 (2016).

5. M. E. MacDonald, A. M. Brandis and B. A. Cruden, 2018 Joint Thermophysics and Heat Transfer Conf. (2018), p. 4067.

6. B. A. Cruden, A. M. Brandis and M. E. MacDonald, 2018 Joint Thermophysics and Heat Transfer Conf. (2018), p. 3768.

7. C. Park, 46th Aerospace Sciences Meeting and Exhibit (2008), AIAA paper 2008-1446.

8. D. F. Potter, Ph.D. thesis, The University of Queensland, Australia (2011).

9. A. Aliat, E. Kustova and A. Chikhaoui, Chem. Phys. Lett. 390, 370 (2004). 
Q. Hong et al.

10. C. O. Johnston, A. M. Brandis and K. Sutton, 43rd AIAA Thermophysics Conf., 2012, AIAA paper 2012-2886.

11. C. W. Gear, Numerical Initial-Value Problems in Ordinary Differential Equations (Prentice-Hall, Englewood Cliffs, NJ, 1971). 